\title{
A High Gain Omnidirectional COCO Antenna Array
}

\author{
Yang $\mathrm{Yu}^{\mathrm{a})}$ and Weiguo Dang ${ }^{\mathrm{b})}$ \\ Postgraduate management group, Engineering University of PAP1,3, Xi'an, Shan xi 710086, China. \\ a)772857324@qq.com \\ b) dangweiguo@qq.com
}

\begin{abstract}
This paper presents a high gain wideband omnidirectional COCO antenna array. The antenna array operates at $1500 \mathrm{MHz}-2000 \mathrm{MHz}$ and can be used for mobile communication. The planar micro-strip array antenna is composed of seventeen trapezoidal micro-strip patch antennas with different sizes. By loading a matching resistance, the antenna can achieve an impedance bandwidth of $28.5 \%$ for $\mathrm{VSWR}<=2$, and it has good horizontal omnidirectional radiation characteristics. The out-of-roundness is less than $0.3 \mathrm{~dB}$ and the highest gain is $9 \mathrm{~dB}$.
\end{abstract}

Key words: COCO; horizontal omnidirectional; out-of-roundness; micro-strip.

\section{INTRODUCTION}

With the rapid development of wireless information technology, the corresponding communication systems are becoming more and more diverse, and the working environment of the mobile communication system is becoming more and more complex. In such a working environment, the quality of communication is seriously declined. In order to achieve better communication quality, the high gain omnidirectional radiation antenna is mostly required, and at the same time the size of the antenna must be small and the cost must be low. Coaxial collinear antenna (COCO antenna) has the advantages of omnidirectional radiation, simple structure, and be easy to form an array. Many communication and radar systems use it to form large array antenna system. COCO antenna was first proposed by American scholars B. B. Balsleyh and W.L. Ecklund and others in 1972. So far, a lot of progress has been made [1, 2]. In Reference [3, 4], a coaxial collinear antenna array model is presented. Each radiation unit is alternately distributed at both ends of the medium plate, and they are connected by the micro-strip line, and each section of the micro-strip line has an impedance matching unit. The Reference [3] consists of six trapezoid radiation units with different areas, working in $1.60 \mathrm{GHz}-1.66 \mathrm{GHZ}$, the relative bandwidth is only $3.6 \%$, the antenna gain is greater than $7 \mathrm{~dB}$, and the maximum gain is $8.6 \mathrm{~dB}$. And Reference [4] consists of 8 units, the relative bandwidth is $4 \%$, and the highest gain is $4.85 \mathrm{~dB}$. In Reference [5], a new type of planar coaxial collinear antenna array is proposed, which is distributed on both sides of the medium plate by two pairs of alternating rectangular coaxial patches as radiation units. The relative bandwidth can reach $24.5 \%$ and the gain is more than $4 \mathrm{~dB}$.

The design of COCO antenna can achieve better omnidirectional radiation characteristics, but the bandwidth is not wide enough, even is rather narrow. In view of the shortcomings of the above design, this paper obviously improves the bandwidth of the antenna array by loading 50 Omega matching resistors at the top of the COCO array and adopting the ladder shaped patch with gradient structure. The feed is fed by the microstrip balance transmission line. Compared with the existing COCO antenna array, the proposed antenna array not only achieves good omnidirectional radiation, but also has a wider frequency band and higher gain. In the whole frequency band, the roundness is less than $0.3 \mathrm{~dB}$, the gain is above $7.65 \mathrm{~dB}$ and the relative bandwidth is $28.6 \%$. 


\section{ANTENNA STRUCTURE AND DESIGN}

\section{The Structure of the Antenna}

The structure and physical properties of COCO antenna array are shown in Figure 1. (a), (b) and (c) are the top, side and bottom view of the antenna array respectively. The antenna is printed on the Rogers RT/duroid 5880 plate with a relative dielectric constant of 2.2 and a thickness of $1 \mathrm{~mm}$, and the whole structure is upright antenna array, whose size is $836.5 \mathrm{~mm} * 28 \mathrm{~mm} * 1 \mathrm{~mm}$. The width of trapezoidal metal patch is gradual, printed on both sides of the dielectric substrate. Adopted micro-strip balanced transmission line feeding mode, the seventeen-element linear array is fabricated. The central frequency of the antenna is $1.8 \mathrm{GHz}$. The length of the ladder shaped patch is L2. The maximum width of the ladder shaped patch is Wmax, the minimum width is Wmin, and the distance between patchs is Ld. The specific parameters of the antenna are as follows in Table 1.

TABLE 1. Geometric parameters of the proposed antenna array

\begin{tabular}{cccccc}
\multicolumn{5}{c}{ TABLE 1. Geometric parameters of the proposed antenna array } \\
\hline $\mathrm{L}$ & $836.5 \mathrm{~mm}$ & $\mathrm{Ld}$ & $49.5 \mathrm{~mm}$ & $\mathrm{~L} 3$ & $49 \mathrm{~mm}$ \\
$\mathrm{~W}$ & $28 \mathrm{~mm}$ & $\mathrm{~L} 1$ & $59 \mathrm{~mm}$ & Wmax & $28 \mathrm{~mm}$ \\
$\mathrm{Wd}$ & $4 \mathrm{~mm}$ & $\mathrm{~L} 2$ & $47.5 \mathrm{~mm}$ & Wmin & $16 \mathrm{~mm}$ \\
\hline
\end{tabular}

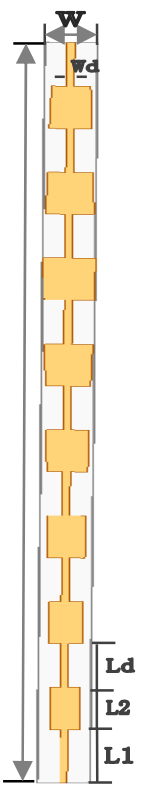

(a)

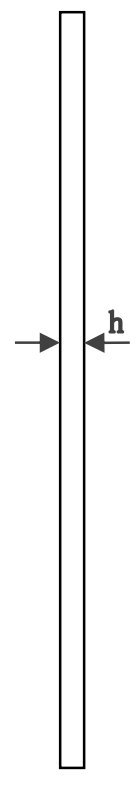

(b)

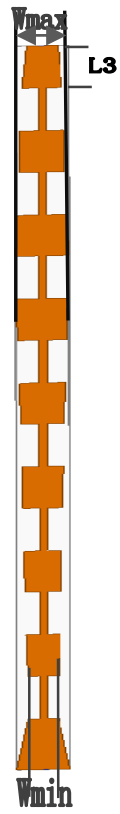

(c)

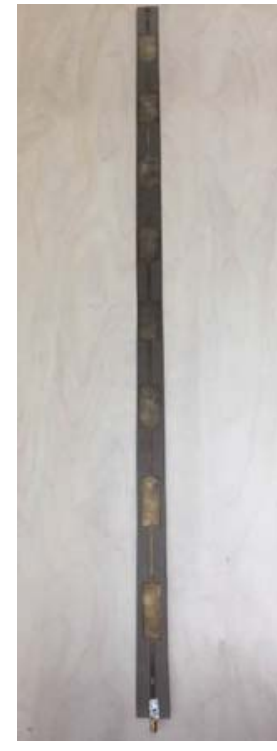

(d)

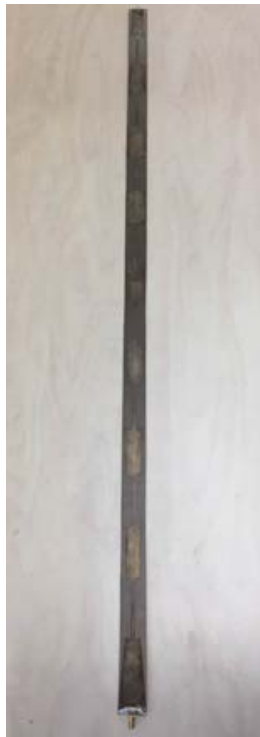

(e)

FIGURE 1. Antenna structure and material object

\section{The Design of Antenna}

In order to reduce sidelobe, metal radiation patch adopts the gradual structure. The radiated patches with the width changed gradually along the balanced transmission line from the middle to the two ends help to make the current evenly distributed on the metal sheet and improve the bandwidth and power capacity of the antenna array. Figure 2 shows the influence of the radiated patchs with gradual width and the radiating plate without gradient structure on the performance of the antenna. Figure 2 (a) is the influential diagram of S11 result with or without gradual structure. Figures 2 (b), (c) and (d) are the comparable diagrams of the influence of gradual structure on the E-plane radiation pattern of low frequency, center frequency and high frequency. It can be seen from the diagrams that the radiation patch with the width gradient can help to expand the bandwidth of the antenna array, improve the radiation characteristics of the antenna array, and can effectively reduce the sidelobe and improve the performance of the antenna array. 


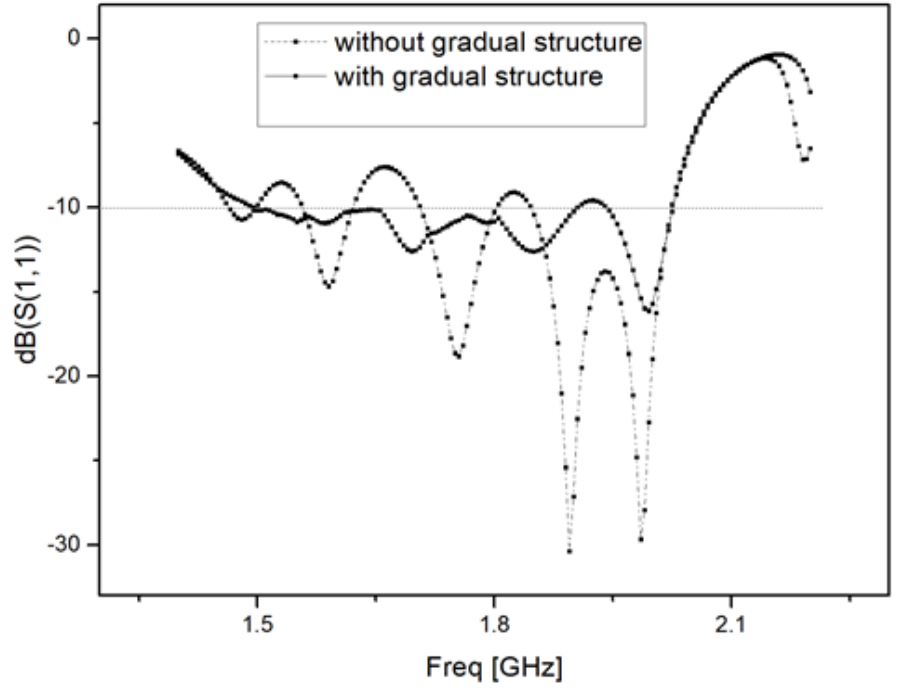

(a)

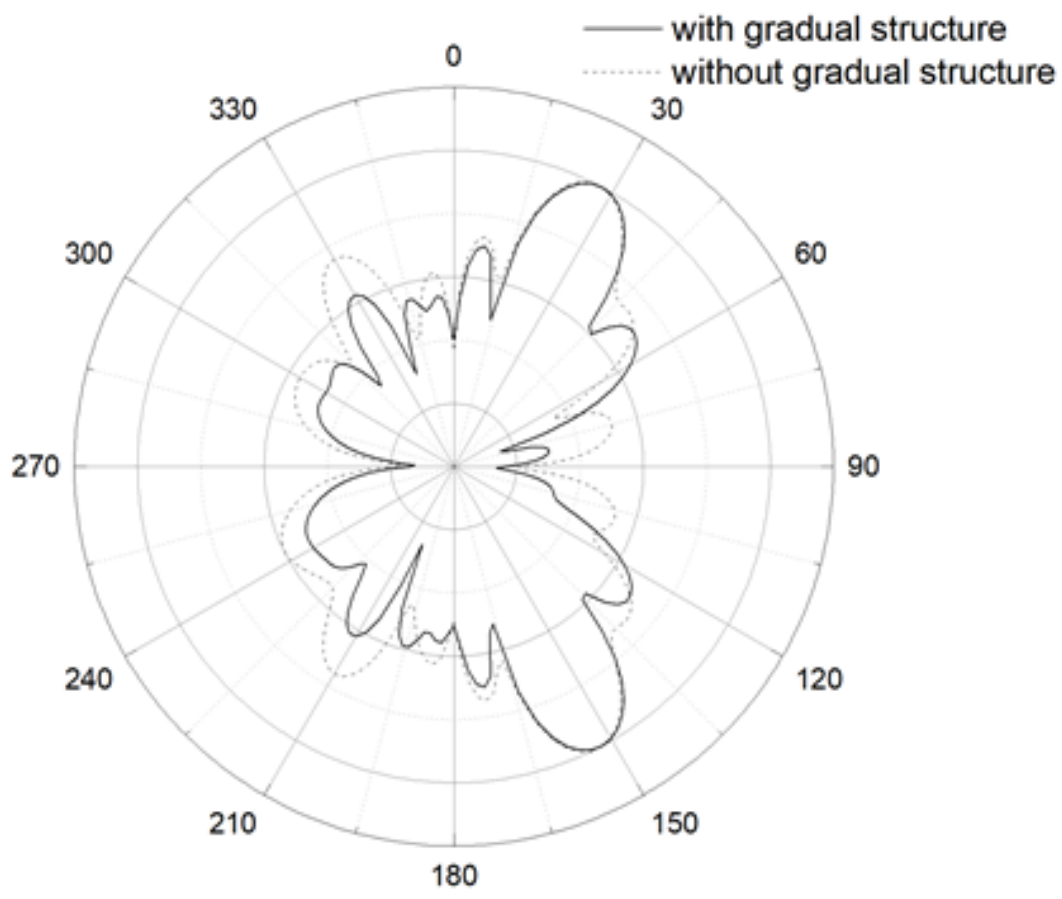

(b) 


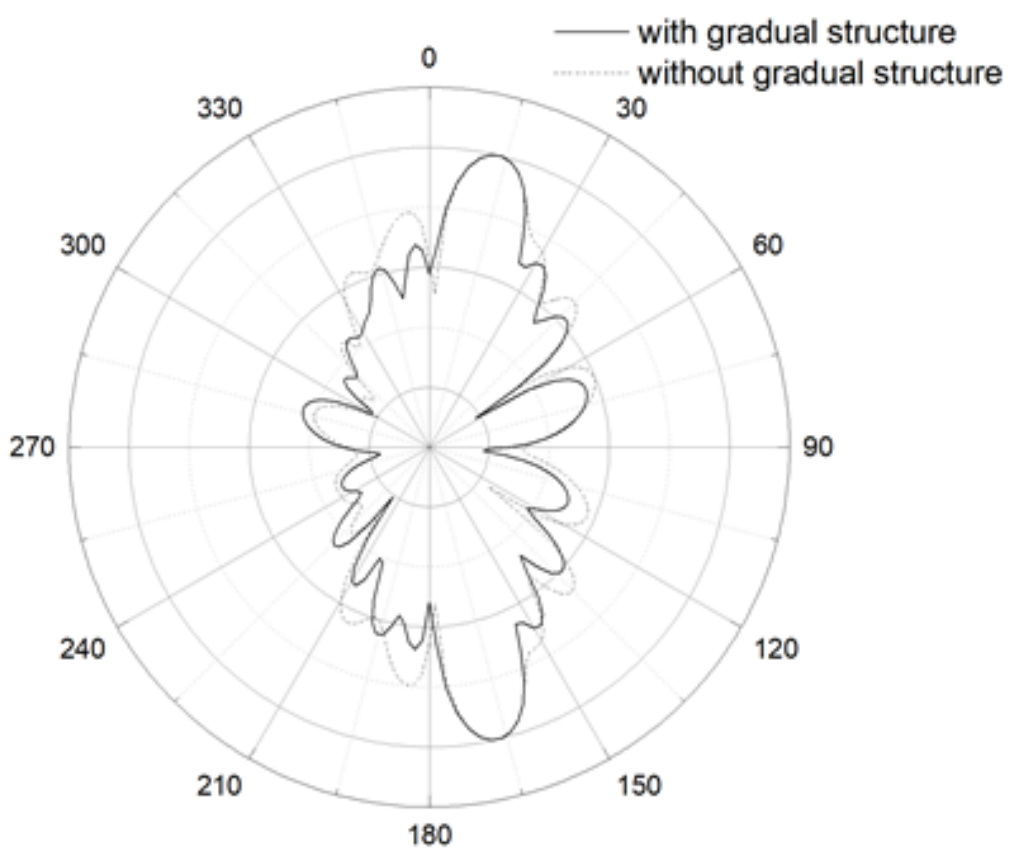

(c)

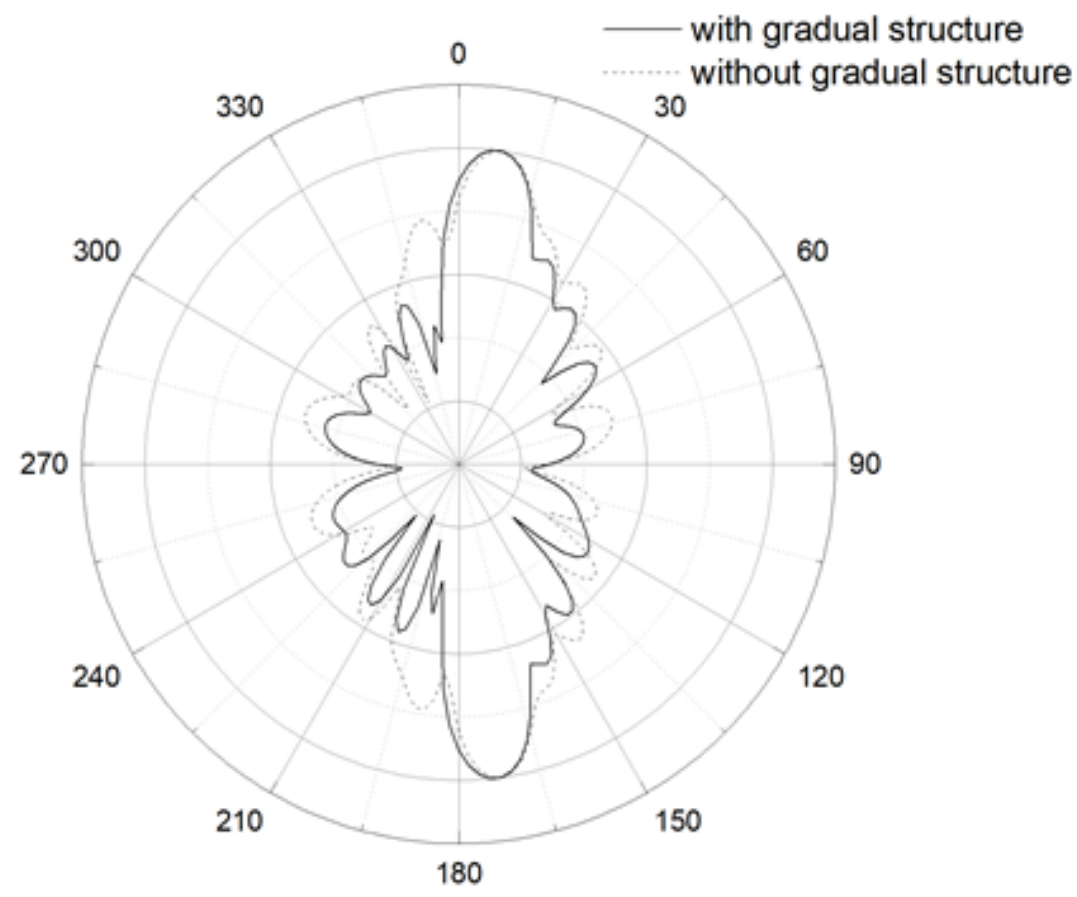

(d)

FIGURE.2. Influence of gradual structure on radiation performance of antenna 


\section{The Optimal Design of Antenna}

Loading the matching resistance at the end of the antenna can improve the current distribution on the antenna surface and make it into traveling wave distribution or similar traveling wave distribution, so that the energy reflected from the inside and the end of the antenna can be reduced, and the band of the antenna can be broadened obviously and the radiation waveform can be improved. That loading resistance at the end of the antenna array not only has fewer load points, but also be far away from the feed point, so it is widely used.

Based on the principle of impedance loading, the resistance is loaded at the top of the transmission line at the end of the antenna, and the micro-strip transmission lines on both sides of the medium plate are connected by the resistance. Figure 3 compares the effects of loading resistance, open circuit and short circuit on $|\mathrm{S} 11|$. Loading a resistance can effectively broaden the bandwidth and improve the radiation performance of the antenna. Figure 4 compares the influence of resistance on antenna gain. It shows that the loading resistance has nearly no significant effect on the radiation efficiency of the antenna. At the high frequency section, the two lines basically coincide, and the gain slightly increases at the low frequency band. This is because the antenna efficiency is not the monotone function of the parameter $\rho$ after loading resistance. It increases first and then decreases in a certain range. After adjusting the geometric parameters of the fixed antenna, the antenna can get the maximum bandwidth and efficiency [9]. Figure 5 compares the effects of resistance with different values in a certain range on the antenna VSWR. The trend of the curves is almost the same. With the increase of the resistance value of the loading resistance, the VSWR is lower, and the frequency response characteristics are improved more obviously. After simulation testing, it is found that when the resistance is $50 \mathrm{ohms}$, the antenna has the best radiation performance, the broadening bandwidth is widest and the gain is highest.

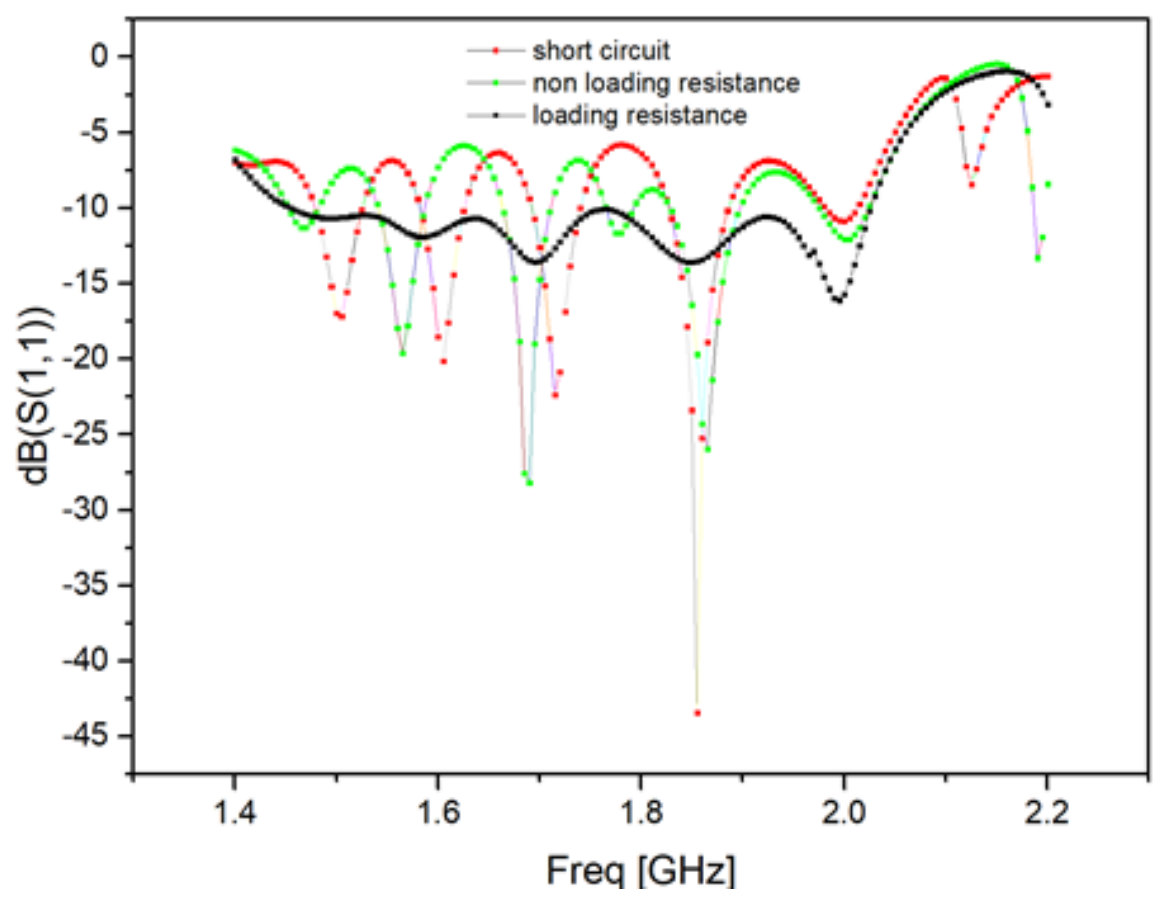

FIGURE.3. The effect of loading resistance, non-loading resistance and short circuit on the antenna S11 


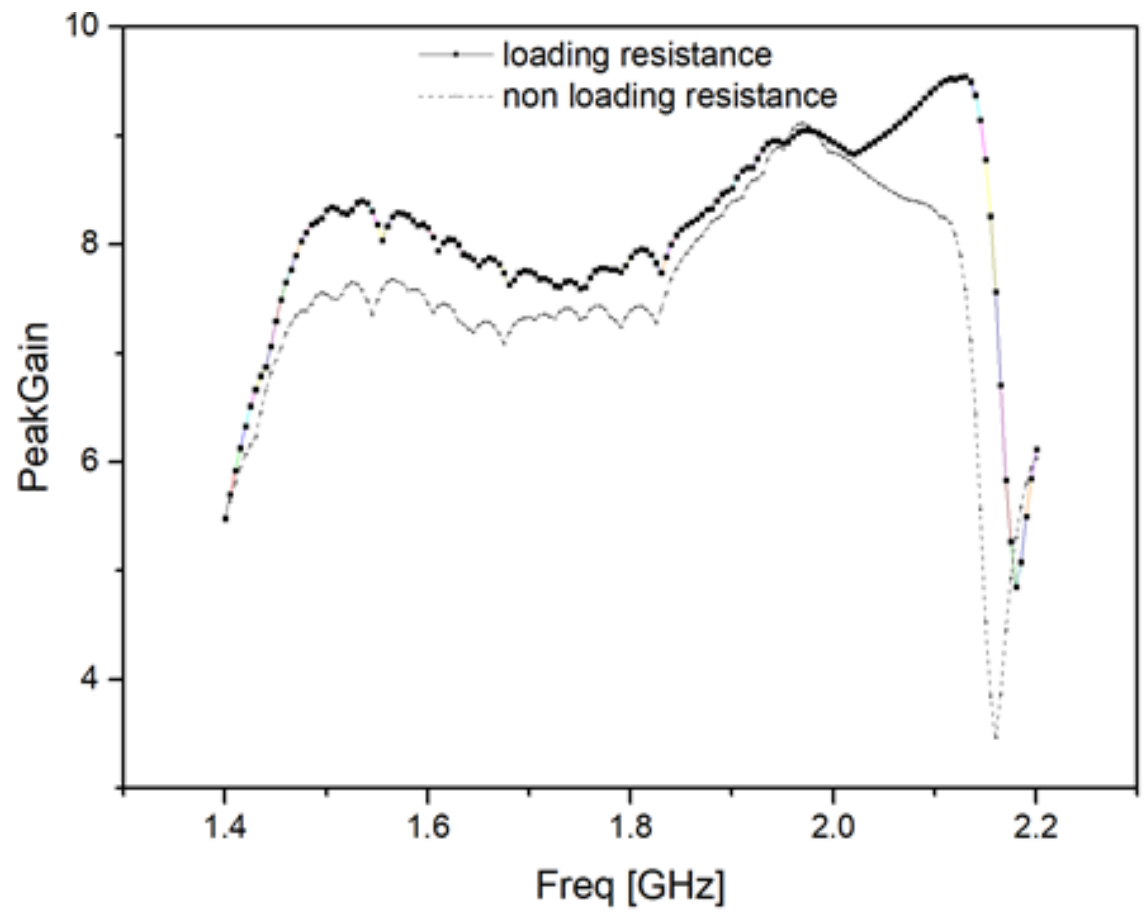

FIGURE.4. Effect of loading resistance and non-loading resistance on antenna gain

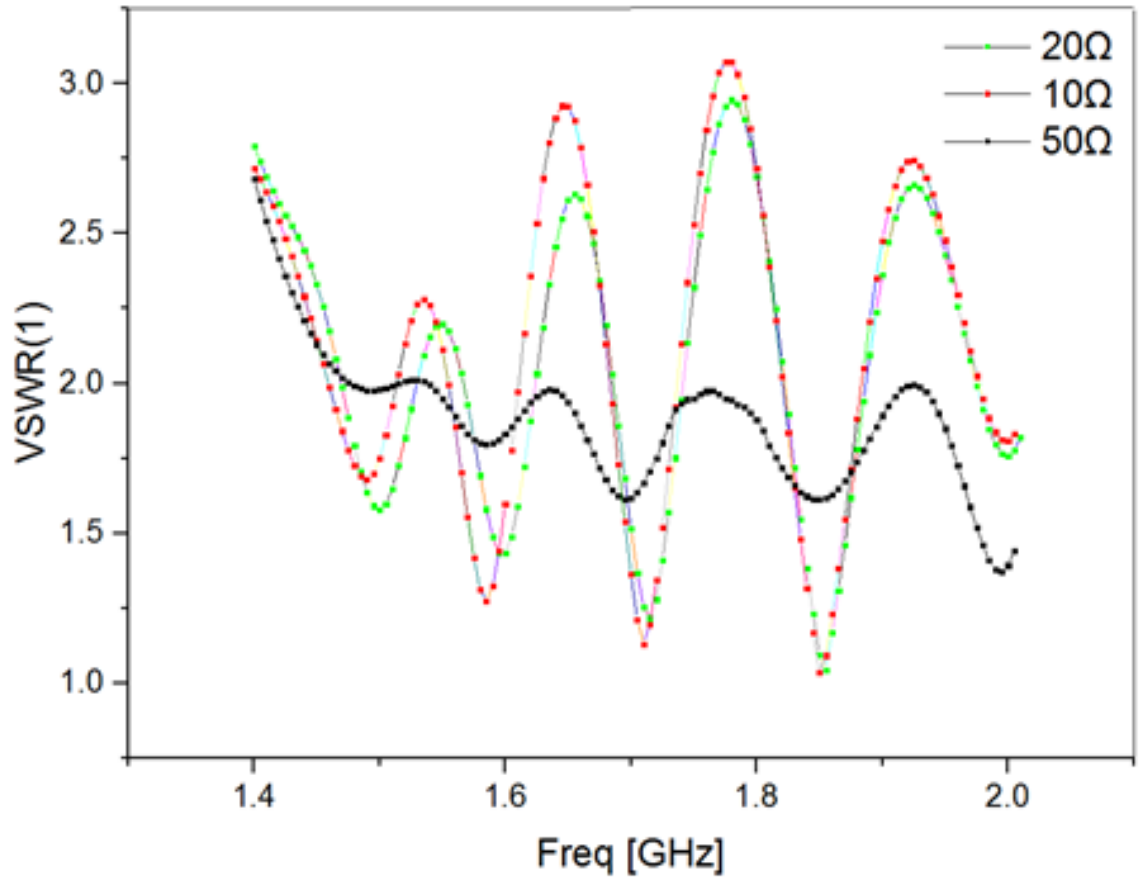

FIGURE.5. Influence of loading of different values on antenna VSWR

\section{MEASURED RESULTS AND ANALYSIS}

As shown in Figure 1 (d) and (E), the object is fabricated according to the simulation optimization model. The vector network analyzer is used to test the physical objects. The simulation results of VSWR test are shown in 
Figure 6. In general, the measured results are in good agreement with the simulation results, and the measured results are better than the simulation results overall, especially at the low frequency segments. Though there is a slightly discrepancy at the high frequency band, it still meets the design requirements. The difference is mainly caused by processing technology, accuracy, testing environment and instability of dielectric constant.

The radiation characteristics and gain of the antenna are tested. The gain of the antenna in the whole frequency band is shown in Figure 7, the highest gain is $9.05 \mathrm{~dB}$, and the minimum gain is $7.62 \mathrm{~dB}$. Figure 8 shows the E-plane and $\mathrm{H}$-plane radiation pattern of the antenna at three frequency points of $1.6 \mathrm{GHz}, 1.8 \mathrm{GHz}$ and $2.0 \mathrm{GHz}$. From the graph, we can see that the E-plane basically presents the omnidirectional radiation in the whole frequency band, and the roundness of the omnidirectional surface is less than $0.3 \mathrm{~dB}$

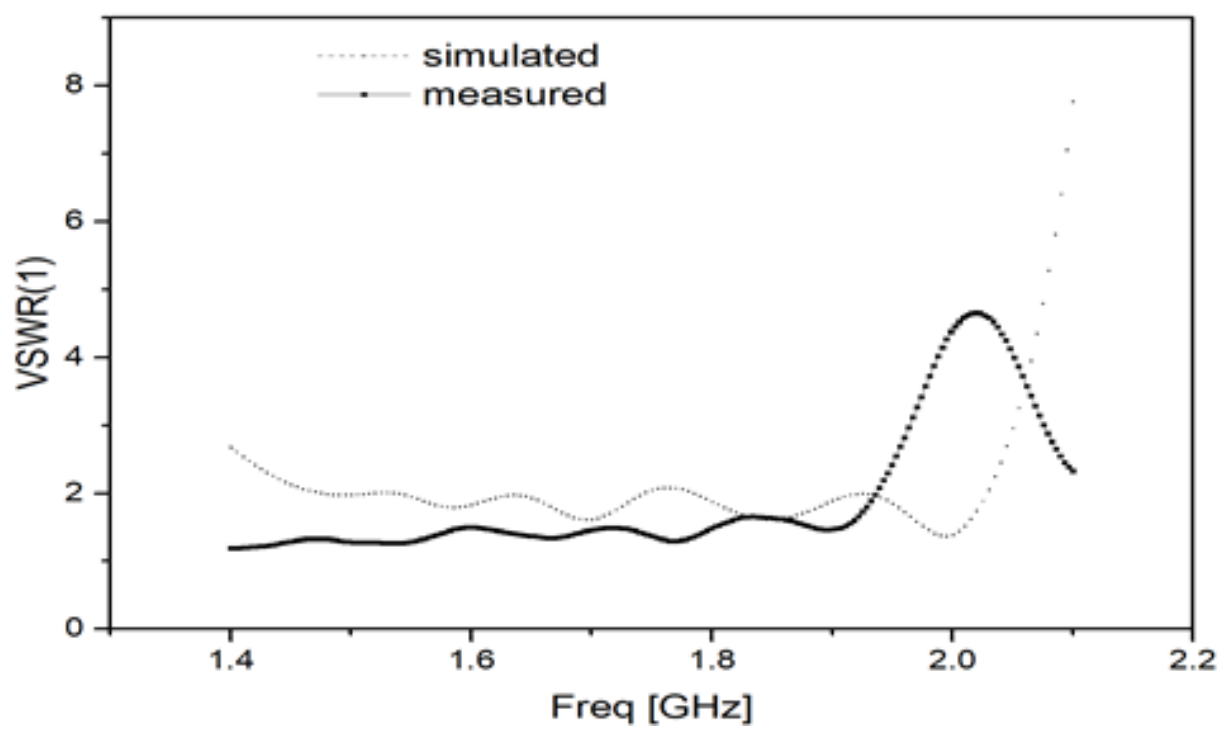

FIGURE.6. Contrast diagram of antenna VSWR test and simulation effect

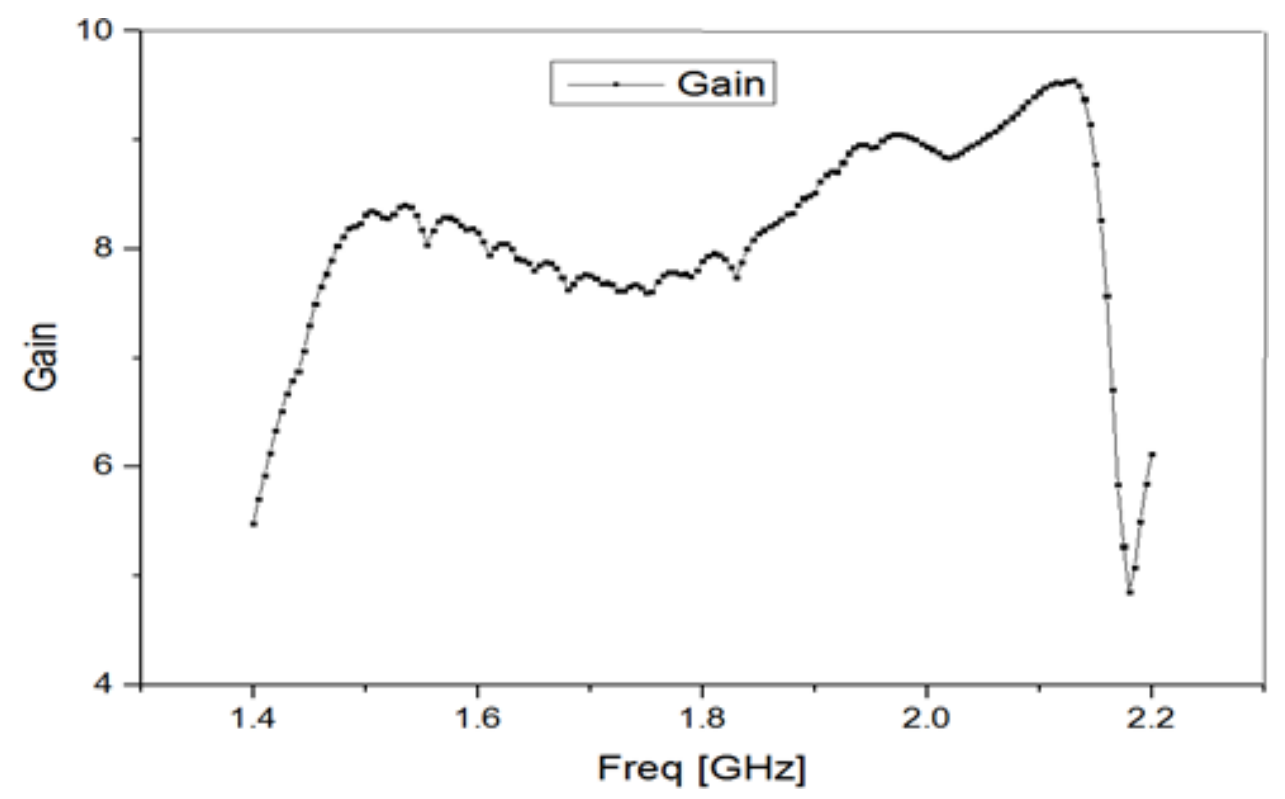

FIGURE.7. Antenna gain 


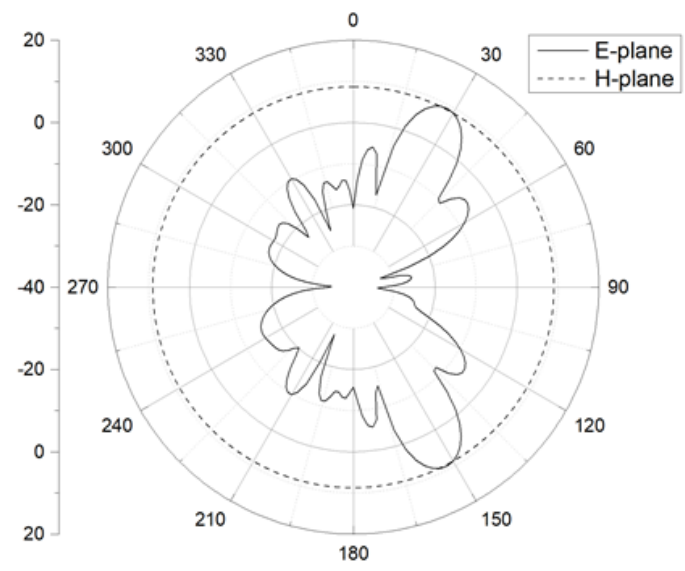

(a)

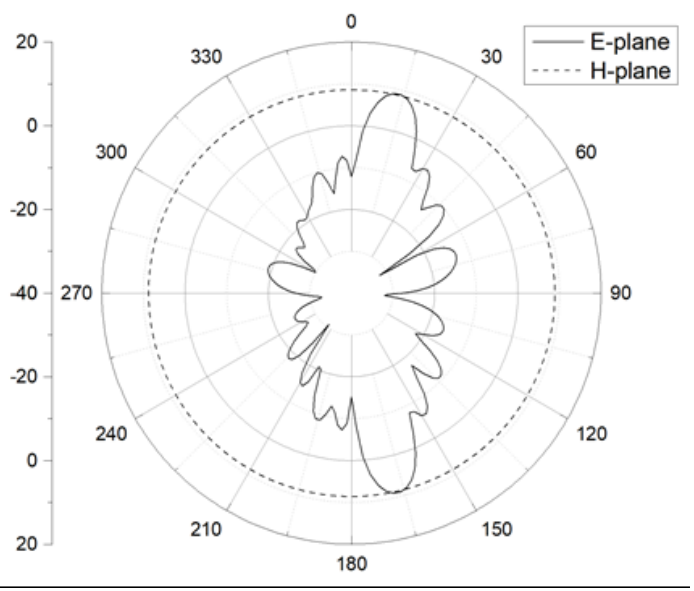

(b)

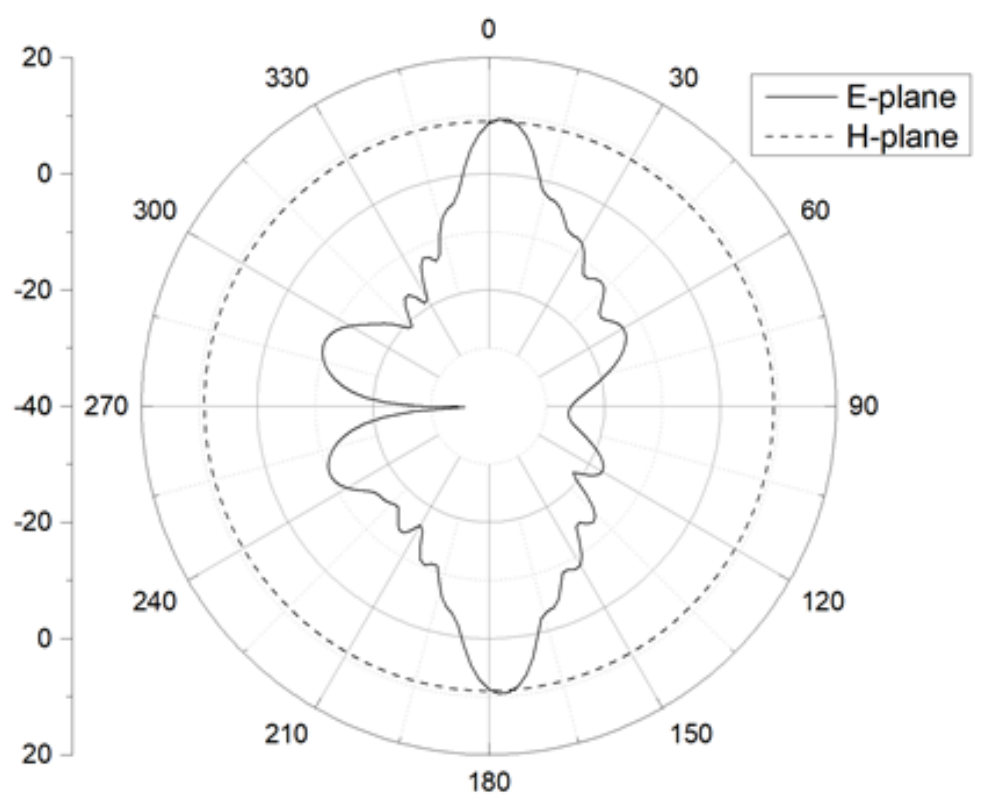

(c)

FIGURE.8. Antenna measurement pattern

\section{CONCLUSION}

A high gain omnidirectional wideband COCO antenna array is designed, which can effectively reduce the sidelobe by using radiation patch with gradient structure, and effectively improve the antenna radiation performance and increase the bandwidth by loading a matching resistance at the end of antenna array. The measured VSWR in the $1500 \mathrm{MHz}-2000 \mathrm{MHz}$ band is less than 2, the antenna pattern is less than $0.3 \mathrm{~dB}$ in the whole frequency band, and the measured gain of the antenna is 7.62 to $9.05 \mathrm{~dB}$. The antenna array is simple in structure and easy to process. It can be widely applied in wireless communication. 


\section{ACKNOWLEDGMENTS}

This work is supported in part by the National Natural Science Foundation of China under Grant 61302051 and in part by the National Natural Science Foundation of China under Grant 61771490. And it is also supported by the Basic Foundation of Engineering University of PAP under Grant WJY201606.

\section{REFERENCES}

1. Balsley B, Ecklund W. “A portable coaxial collinear antenna,” IEEE Transactions on Antennas \& Propagation. 1972, pp: 513-516.

2. Judasz T, Ecklund W, Balsley B. "The coaxial collinear antenna: Current distribution from the cylindrical antenna equation,” IEEE Transactions on Antennas \& Propagation. 1987, pp: 327-331.

3. Tang J, Fang L, Cheng H. "A low sidelobe and high gain omni-directional COCO antenna array," in 3rd AsiaPacific Conference on Antennas and Propagation, 2014, pp: 339-341.

4. Zhao P F, Si L M, Liu Y, et al. "A novel omnidirectional microstrip coaxial collinear antenna array," in International Radar Conference,2013, pp:1-4

5. Wang J, Liu X, Yang X, et al. "A planar Coaxial Collinear antenna with rectangular coaxial strip," in International Symposium on Atennas \& Propagation, 2014, pp:716-719

6. Wang Xinbao, "Research on high gain omnidirectional antenna," Master's thesis, Xi'an Electronic and Science University, 2013.

7. Zhong Shunshi, Antenna theory and technology, The electronic industry press, Beijing,2015, pp:198-200.

8. Tang Yan, Cao Zhenxin, "A highly efficient and highly impedance omnidirectional COCO antenna," Journal of microwave. 2012, pp: 86-89.

9. Guo Yuchun, "the history of David research on efficient resistive loading antenna theory," Journal of radio science. 2007, pp: 276-280. 